\title{
An extension of Zassenhaus' theorem on endomorphism rings
}

\author{
by \\ Manfred Dugas (Waco, TX) and Rüdiger Göbel (Essen)
}

\begin{abstract}
Let $R$ be a ring with identity such that $R^{+}$, the additive group of $R$, is torsion-free. If there is some $R$-module $M$ such that $R \subseteq M \subseteq \mathbb{Q} R\left(=\mathbb{Q} \otimes_{\mathbb{Z}} R\right)$ and $\operatorname{End}_{\mathbb{Z}}(M)=R$, we call $R$ a Zassenhaus ring. Hans Zassenhaus showed in 1967 that whenever $R^{+}$is free of finite rank, then $R$ is a Zassenhaus ring. We will show that if $R^{+}$is free of countable rank and each element of $R$ is algebraic over $\mathbb{Q}$, then $R$ is a Zassenhaus ring. We will give an example showing that this restriction on $R$ is needed. Moreover, we will show that a ring due to A. L. S. Corner, answering Kaplansky's test problems in the negative for torsion-free abelian groups, is a Zassenhaus ring.
\end{abstract}

1. Introduction. In 1963 A. L. S. Corner [5] proved his celebrated result that any countable, torsion-free, reduced ring is the endomorphism ring of a countable torsion-free, reduced abelian group. Moreover, he was able to show that if $R$ is such a ring of finite rank $n$, then there exists an abelian group $A$ such that $\operatorname{End}(A)=R$ and $A$ has rank $2 n$. He also gave an example of a ring $R$ of rank $n$ that is not the endomorphism ring of any group of rank less than $2 n$. This makes it natural to ask for which torsion-free rings $R$ of rank $n$ there exists an abelian group $A$ of the same rank $n$ such that $\operatorname{End}(A)=R$. An answer was obtained by H. Zassenhaus [15] in 1967: If $R$ is a ring with identity such that $R^{+}$, the additive group of $R$, is free of finite rank, then there exists an $R$-module $M$ such that $R \subseteq M \subseteq \mathbb{Q} R$ and $\operatorname{End}(M)=R$. Of course, the first clause means that $R$ and $M$ have the same rank. In 1968, M. C. R. Butler [4], using similar techniques to those in [15], extended this result to finite rank rings $R$ such that $R_{p}$, the localization of

2000 Mathematics Subject Classification: Primary 20K20, 20K30; Secondary 16S60, $16 \mathrm{~W} 20$.

Key words and phrases: endomorphism rings, Zassenhaus' theorem.

Support through a sabbatical leave granted by Baylor University in the spring of 2006 and the hospitality of the Universität Duisburg-Essen are gratefully acknowledged.

This work is supported by the project No. I-706-54.6/2001 of the German-Israeli Foundation for Scientific Research \& Development. 
$R^{+}$at the prime $p$, is a free $\mathbb{Z}_{p}$-module for all primes $p$. We introduce the following

Definition 1. Let $R$ be a ring with $1 \in R$ such that $R^{+}$is torsion-free. If there exists an $R$-module $M$ such that $R \subseteq M \subseteq \mathbb{Q} R$ and $\operatorname{End}_{\mathbb{Z}}(M)=R$, then we call $R$ a Zassenhaus ring with module $M$.

Recently, Zassenhaus rings made a somewhat surprising appearance in connection with quasi-localizations: Extending the well-known notion of a localization (see e.g. [13, p. $462 \mathrm{ff}$.$] ), a homomorphism \alpha: A \rightarrow B$ is called a quasi-localization of $A$ if for all homomorphisms $\phi: A \rightarrow B$ there exists some natural number $n$ and a unique $\psi \in \operatorname{End} B$ such that $n \phi=\alpha \psi$. It was shown in [1] that an injective homomorphism $\alpha: \mathbb{Z} \rightarrow M$ is a quasilocalization (equivalently, a localization in the quasi-category of torsion-free abelian groups) if and only if there is a Zassenhaus ring $R$ with module $M$.

On the one hand, several new examples of Zassenhaus rings of finite as well as infinite rank were presented in [1] and [2]. We will give an example to show that not all rings $R$ such that $R^{+}$is free of countable rank are Zassenhaus rings. On the other hand, we will obtain the following extension of Zassenhaus' theorem.

THEOREM 1. Let $R$ be a ring with identity such that $R^{+}$is free of countable rank. If each element of $R$ is algebraic over $\mathbb{Q}$, then $R$ is a Zassenhaus ring.

A. L. S. Corner introduced rings $L$ for the Leavitt theorem (cf. [13, Section 15.1]) that lead to negative answers to the Kaplansky test problems in the category of torsion-free abelian groups, rendering this class hopeless for classification. The same holds for $\aleph_{1}$-separable groups (cf. [10]). We will show that these rings $L$ are Zassenhaus rings. This shows that Kaplansky test problems have a negative answer also in the class of torsion-free quasilocalizations of $\mathbb{Z}$.

2. Zassenhaus' result for rings of infinite rank. We will consider rings $R$ such that $R^{+}$is free abelian of any rank. We will always identify elements $r \in R$ with their action on $R$ by right multiplication, i.e. $r(x)=x r$ for all $x \in R$. We begin with a well-known fact but we include a proof for the convenience of the reader.

Proposition 1. Let $f(x) \in \mathbb{Z}[x]$ and $a_{1}(x) \in \mathbb{Q}[x]$ be monic polynomials and $a_{2}(x) \in \mathbb{Q}[x]$ such that $f(x)=a_{1}(x) a_{2}(x)$. Then $a_{i}(x) \in \mathbb{Z}[x]$ for $i=$ 1,2 .

Proof. Note that $a_{2}(x)$ is monic as well. Consider the ideals $\{z \in \mathbb{Z}$ : $\left.z a_{i}(x) \in \mathbb{Z}[x]\right\}=\alpha_{i} \mathbb{Z}$. It is easy to see that $\alpha_{i} a_{i}(x) \in \mathbb{Z}[x]$ is primitive 
for $i=1,2$. Then $\alpha_{1} \alpha_{2} f(x)=\left(\alpha_{1} a_{1}(x)\right)\left(\alpha_{2} a_{2}(x)\right)$ is primitive by Gauss' lemma, which means $\alpha_{1} \alpha_{2} \in\{1,-1\}$ and thus $a_{i}(x) \in \mathbb{Z}[x]$.

We will introduce some notations which will be used throughout this section.

Let $F$ be a free abelian group and $\widetilde{F}=\mathbb{Q} F$ the corresponding $\mathbb{Q}$-vector space. If $\tau \in \operatorname{End}(F)$, then $\tau$ has a unique extension $\widetilde{\tau}: \widetilde{F} \rightarrow \widetilde{F}$.

We say that $\tau \in \operatorname{End}(F)$ is algebraic if there is a non-trivial polynomial $g(x) \in \mathbb{Q}[x]$ such that $g(\tau)=0$.

Let $0 \neq e \in F$ and $V=e \mathbb{Q}[\tau], g(\tau)=0$. Then $V$ is the $\tau$-invariant subspace (of dimension $\leq \operatorname{deg}(g)$ ) of $\widetilde{F}$ generated by $e$, and $W_{*}:=V \cap F$ is a pure, $\tau$-invariant subgroup of $F$. Since $W_{*}$ is a finite rank subgroup of the free group $F$, the group $W_{*}$ is finitely generated and free. Consider the ascending chain $C_{j}=\sum_{i=0}^{j} e \tau^{i} \mathbb{Z} \subseteq W_{*}$. Since $W_{*}$ is Noetherian, there is some $k$ such that $\tau^{k} \in C_{k-1}$. This shows that there is some monic $f(x) \in \mathbb{Z}[x]$ of minimal degree such that $f(\tau)=0$. Let $m(x) \in \mathbb{Q}[x]$ be the (monic) minimal polynomial of $\widetilde{\tau} \mid V$. Then $f(x)=m(x) h(x)$ for some $h(x) \in \mathbb{Q}[x]$. By Proposition $1, m(x) \in \mathbb{Z}[x]$ and it follows that $m(x)=f(x)$ and $k=$ $\operatorname{dim}(V)=\operatorname{rank}\left(W_{*}\right)$. Let $W=\bigoplus_{i=0}^{k-1} e \tau^{i} \mathbb{Z}$. Then $W_{*}$ is the purification of $W$ in $F$.

We have shown the following:

Claim 1. Let $\tau \in \operatorname{End}(F)$ be algebraic and $0 \neq e \in F$. Then there is some $n \in \mathbb{N}$ such that $W:=e \mathbb{Z}[\tau]=\bigoplus_{i=0}^{n-1} e \tau^{i} \mathbb{Z}$ is free abelian of rank $n$ and the minimal polynomial of $\tau\lceil W$ is monic with integer coefficients. The same holds for the minimal polynomial of $\tau$. Let $W_{*}$ be the purification of $W$ in $F$. Then there exists a unique, least natural number $k$ such that $k W_{*} \subseteq W$.

We still use our notations in the next

Claim 2. Let $c \in \mathbb{Z}$ be a non-eigenvalue of $\tau$ and $z \in \mathbb{Z}$. Then ze $\in$ $F(c-\tau)$ implies that $\operatorname{det}((c-\tau)\lceil W)$ divides $k z$.

Proof. There exists $g(x) \in \mathbb{Z}[x]$ of minimal positive degree such that $g(c-\tau)=0$. If $g(0)=0$, then $g(x)=x h(x)$ and it follows that $0=$ $g(c-\tau)=(c-\tau) h(c-\tau)$, and $h(c-\tau) \neq 0$ by the minimality of $\operatorname{deg}(g(x))$; moreover, each non-zero element in $F h(c-\tau) \neq\{0\}$ is an eigenvector of $\tau$ with eigenvalue $c$. This shows that, by the choice of $c$, we have $g(0) \neq 0$. Solving the equation $g(c-\tau)=0$ for the constant term, setting $q=g(0)$, we find that $q(c-\tau)^{-1} \in \mathbb{Z}[c-\tau] \subseteq \mathbb{Z}[\tau]$ and thus $e q(c-\tau)^{-1} \in W$.

Now assume $z \in \mathbb{Z}$ is such that $z e \in F(c-\tau)$.

Then $z e(c-\tau)^{-1} \in F \cap(1 / q) W \subseteq F \cap \mathbb{Q} W=W_{*}$. It follows that $z e \in W_{*}(c-\tau)$ and thus $k z e \in k W_{*}(c-\tau) \subseteq W(c-\tau)$. We infer that there are $z_{i} \in \mathbb{Z}$ for $0 \leq i \leq n-1$ such that $k z e=\left(\sum_{i=0}^{n-1} e z_{i} \tau^{i}\right)(c-\tau)$. Define $h(x)=-k z+\sum_{i=0}^{n-1} z_{i} x^{i}(c-x) \in \mathbb{Z}[x]$ and note that $e h(\tau)=0$. Now the 
(monic) characteristic polynomial $\chi(x)$ of $\tau \uparrow W$ is the same as the (monic) minimal polynomial of $\tau\lceil W$ and has degree $n$. This implies that $h(x)=$ $z_{n-1} \chi(x)$, so that $-k z=h(c)=z_{n-1} \chi(c)$ and thus $\chi(c)=\operatorname{det}((c-\tau)\lceil W)$ divides $k z$.

We will need the following

Lemma 1. Let $A$ be a torsion-free ring with $1 \in A$, and $\mathcal{F}=\left\{P_{i}=\right.$ $\left.b_{i} A \mid i<\omega\right\}$ a countable family of principal right ideals of $A$ such that the $b_{i}$ are not zero-divisors. Moreover, suppose there exist distinct prime integers $p_{i}$ and $d_{i} \in \mathbb{Z}, r_{i} \in \mathbb{N}$ such that $p_{i}^{r_{i}} d_{i} A \subseteq P_{i}$ and $\operatorname{gcd}\left(p_{i}, d_{i}\right)=1$. Let $M=A+\sum_{i<\omega} p^{-r_{i}} P_{i} \subseteq \mathbb{Q} A$. If $y \in M$ is such that right multiplication by $y$ is an endomorphism of $M$, then $y \in A$.

Proof. Since the $p_{i}$ are distinct, $t_{p_{i}}(M / A)=\left(p_{i}^{-r_{i}} P_{i}+A\right) / A$ and $y=v / k$ for some $v \in A$ and $k=\prod_{i \in I_{y}} p_{i}^{s_{i}}$ for some $s_{i} \in \mathbb{N}$ and some finite subset $I_{y}$ of $\omega$. By induction over $e=\sum_{i \in I_{y}} s_{i}$ we will show that $y \in A$. To get started, assume that $e=1$, i.e. $k=p_{i}$ for some $i<\omega$. Then

$$
\left(p_{i}^{-r_{i}} P_{i} y+A\right) / A=\left(p_{i}^{-r_{i}} P_{i} \frac{v}{p_{i}}+A\right) / A \subseteq t_{p_{i}}(M / A)=\left(p_{i}^{-r_{i}} P_{i}+A\right) / A
$$

and we have $p_{i}^{-r_{i}-1} P_{i} v \subseteq p_{i}^{-r_{i}} P_{i}+A$. Multiplying this inclusion by $p_{i}^{r_{i}} d_{i}$ we get $p_{i}^{-1} d_{i} P_{i} v \subseteq d_{i} P_{i}+p_{i}^{r_{i}} d_{i} A \subseteq P_{i}$ by our hypothesis. Thus $d_{i} P_{i} v \subseteq p_{i} P_{i}$ and $P_{i} v \subseteq p_{i} P_{i}$ since $\operatorname{gcd}\left(p_{i}, d_{i}\right)=1$. This implies that $b_{i} v=p_{i} b_{i} a$ for some $a \in A$ and so $b_{i}\left(v-p_{i} a\right)=0$. Since $b_{i}$ is not a zero-divisor in $A$, we infer $v-p_{i} a=0$ and it follows that $y=v / p_{i} \in A$.

The rest of the induction is now easy. Consider $\widetilde{y}=p_{i}^{-1} k y$ for some $i \in I_{y}$. By the above, we find that $\widetilde{y} \in A$ and thus $p_{i} \widetilde{y} \in A$. Now we can use the induction hypothesis and conclude that $y \in A$.

We are now ready to prove the main result of this section.

Theorem 2. Let $A$ be a ring with $1 \in A$ such that $A^{+}$, the additive group of $A$, is free of at most countable rank and each $a \in A$ is algebraic over $\mathbb{Q}$. Then $A$ is a Zassenhaus ring.

Proof. Let

$$
\Sigma=\left\{\sigma \mid \sigma \in \operatorname{Hom}(D, A), D \text { of finite rank, } 1 \in D \subseteq_{*} A^{+}, \sigma(1)=0 \neq \sigma\right\},
$$

where " $\subseteq_{*}$ " denotes a pure subgroup. Clearly any $D$ in $\Sigma$ is a summand of the free group $A^{+}$because its rank is finite. Note that the set $\Sigma$ is countable and we choose an enumeration $\Sigma=\left\{\sigma_{i} \mid i<\omega\right\}$ where $D_{i}$ is the domain of $\sigma_{i}$. By induction over $i<\omega$ we choose:

(1) $a_{i} \in D_{i}$ with $e_{i}=-a_{i} \sigma_{i} \neq 0$. This can be done since $\sigma_{i} \neq 0$. 
(2) $W_{i}:=e_{i} \mathbb{Z}\left[a_{i}\right]$ and $k_{i} \in \mathbb{N}$ such that $k_{i}\left(W_{i}\right)_{*} \subseteq W_{i}$. Moreover, $c_{i}$ is not an eigenvalue of $a_{i}$. (Here we identify $a_{i} \in A$ with right multiplication by $a_{i}$.) Let $f_{i}(x)$ be the monic, integer minimal polynomial of $a_{i} \uparrow W_{i}$.

(3) Finally, choose distinct prime integers $p_{i}, c_{i} \in \mathbb{Z}$, and $r_{i}, d_{i} \in \mathbb{N}$ with $\operatorname{gcd}\left(d_{i}, p_{i}\right)=1$ such that $d_{i} e_{i} \notin\left(c_{i}-a_{i}\right) A$ and $p^{r_{i}} d_{i} A \subseteq\left(c_{i}-a_{i}\right) A$.

Assume all the above data have been defined for $j=0,1, \ldots, i-1$. By a result in [3] (see also [13, p. 407, Proposition 12.1.5]), the set

$N_{i}=\left\{q \mid q\right.$ a prime such that $f_{i}(x) \bmod q$ has a root in $\left.\mathbb{Z} / q \mathbb{Z}\right\}$

is infinite. Now simply choose $p_{i} \in N_{i}-\left\{p_{0}, p_{1}, \ldots, p_{i-1}\right\}$ such that $p_{i}$ does not divide $k_{i}$, and since $f_{i}(c) \equiv 0 \bmod p_{i}$ has infinitely many solutions in $\mathbb{Z}$, we may pick a solution $c_{i}$ that is not an eigenvalue of $a_{i}$ and also not a root of $f_{i}(x)$. Let $f_{i}\left(c_{i}\right)=p_{i}^{r_{i}} d_{i}$ with $r_{i}>0$ and $\operatorname{gcd}\left(p_{i}, d_{i}\right)=1$. We have seen above that there is some $a_{i} \in A$ such that $\left(c_{i}-a_{i}\right) a=p_{i}^{r_{i}} d_{i} 1$ and $p_{i}^{r_{i}} d_{i} A \subseteq\left(c_{i}-a_{i}\right) A$. Moreover, $d_{i}\left(c_{i}-a_{i}\right) \sigma_{i}=-d_{i}\left(a_{i} \sigma_{i}\right)=d_{i} e_{i} \notin\left(c_{i}-a_{i}\right) A$ since $f_{i}\left(c_{i}\right)=p_{i}^{r_{i}} d_{i}$ does not divide $d_{i} k_{i}$.

Now set $P_{i}=\left(c_{i}-a_{i}\right) A$ and define the right $A$-module

$$
M=A+\sum_{i<\omega} p_{i}^{-r_{i}} P_{i} \subseteq \mathbb{Q} A
$$

and consider $\varphi \in \operatorname{End}(M)$. Then there is some $n \in \mathbb{N}$ such that $n \varphi(1) \in A$ and define $\sigma=n \varphi-n \varphi(1)$. Note that $\sigma(1)=0$.

CASE 1: $\sigma=0$. In this case, $\varphi=\varphi(1) \in M$ is right multiplication by the element $\varphi(1) \in M$. By Lemma 1 , we have the desired result $\varphi=\varphi(1) \in A$.

CASE 2: $\sigma \neq 0$. We will show that this case does not occur. Let $D$ be a finite rank, pure subgroup of $A^{+}$such that $1 \in D$ and $\sigma\lceil D \neq 0$. Then there are some $i<\omega$ and $\ell \in \mathbb{N}$ such that $D=D_{i}$ and $\ell \sigma \mid D=\sigma_{i} \in \Sigma$ since $D_{i}$ is finitely generated. By construction, there exist $a_{i} \in D_{i}$ and $c_{i} \in \mathbb{Z}$ such that $\ell\left(-a_{i}\right) \sigma=\left(-a_{i}\right) \sigma_{i}=e_{i} \notin\left(c_{i}-a_{i}\right) A=P_{i} \supseteq p_{i}^{r_{i}} d_{i} A$. Let $D_{i}^{*}$ denote the purification of $D_{i}$ in $M$. Since $\sigma_{i}: D_{i} \rightarrow A$, it follows that $\sigma_{i}$ induces a map $\widetilde{\sigma}_{i}$ from $D_{i}^{*} / D_{i}$ into $M / A$. In particular, $\widetilde{\sigma}_{i}$ maps $t_{p_{i}}\left(D_{i}^{*} / D_{i}\right)=\left(\left(p_{i}^{-r_{i}} P_{i} \cap D_{i}^{*}\right)+D_{i}\right) / D_{i}$ into $t_{p_{i}}(M / A)=\left(p_{i}^{-r_{i}} P_{i}+A\right) / A$ and we infer that $d_{i}\left(P_{i} \cap p^{r_{i}} D_{i}^{*}\right) \sigma_{i} \subseteq P_{i} d_{i}+p^{r_{i}} d_{i} A \subseteq P_{i}$ and $c_{i}-a_{i} \in P_{i} \cap p^{r_{i}} D_{i}^{*}$ and so we get the contradiction $d_{i}\left(c_{i}-a_{i}\right) \sigma_{i}=d_{i}\left(-a_{i}\right) \sigma_{i}=d_{i} e_{i} \in P_{i}$, i.e. Case 2 does not occur. This shows that $\operatorname{End}(M)=A$.

Corollary 1. Let $\mathbb{Q}^{c}$ be the algebraic closure of $\mathbb{Q}$ in the field of complex numbers. Then any subring $S$ of $\mathbb{Q}^{\mathrm{c}}$ such that $S^{+}$is free is a Zassenhaus ring. Moreover, $S$ is a subring of the ring of algebraic integers of $\mathbb{Q}^{c}$.

In the above theorem, we have to restrict ourselves to rings whose additive group is free of at most countable rank since there are only countably many prime integers. We can allow rings with free additive groups in the 
following, weaker result. Let $A$ be any ring and $\mathcal{R}_{A}$ the family of all right ideals of $A$ and $\operatorname{End}\left(A, \mathcal{R}_{A}\right)=\left\{\varphi \in \operatorname{End}\left(R^{+}\right) \mid J \varphi \subseteq J\right.$ for all $\left.J \in \mathcal{R}_{A}\right\}$. We will show that all rings with free additive group and algebraic over $\mathbb{Q}$ have "enough right ideals".

Theorem 3. Let $A$ be a ring with identity and with free additive group such that all elements of $A$ are algebraic over $\mathbb{Q}$. Then $\operatorname{End}\left(A, \mathcal{R}_{A}\right)=A$.

Proof. We will employ ideas and results from the previous proof. Let $a \in A, 0 \neq e \in A$, and $W=e \mathbb{Z}[a]$. As we have seen above, the element $a \uparrow W$ has a monic minimal polynomial $f(x) \in \mathbb{Z}[x]$. Let $W_{*}=\mathbb{Q} W \cap A$ be the purification of $W$ in $A$. Then, as we have seen, there exists some $k_{W} \in \mathbb{N}$ such that $k_{W} W_{*} \subseteq W$. Let $c \in \mathbb{Z}$ be such that $c$ is not an eigenvalue of $a$, which we identify with the right multiplication by $a$. We may also assume that $f(c) \neq 0$. Let $z \in \mathbb{Z}$. We have seen that $z e \in(c-a) A$ implies that $f(c)$ divides the product $z k_{W}$. Choose a prime $q$ such that $f(c) \equiv 0 \bmod q$. We have seen that there are infinitely many primes $q$ and integers $c$ to choose from. Thus we may assume that $f(c)=q^{r} d$ and $q$ divides neither $d$ nor $k_{W}$. Since $c$ is no eigenvalue of $a$ we again obtain $q^{r} d A \in(c-a) A$ but de $\notin(c-a) A$, since $q$ divides $f(c)$ but not $d k_{W}$, which implies that $f(c)$ does not divide $d k_{W}$. Let $\kappa$ denote the cardinality of $A$ and

$$
\Sigma=\left\{\sigma \in \operatorname{Hom}(D, A) \mid D \sqsubseteq A^{+} \text {of finite rank, } 1 \in D \text { and } \sigma(1)=0 \neq \sigma\right\} .
$$

Since this set has cardinality $\kappa$ we may label $\Sigma=\left\{\sigma_{i} \mid i<\kappa\right\}$. For each $i<\kappa$ we pick $a_{i} \in D_{i}$ and $c_{i} \in \mathbb{Z}$ such that $\sigma_{i}\left(a_{i}\right)=e_{i} \notin\left(c_{i}-a_{i}\right) A=: J_{i}$. Now let $\varphi \in \operatorname{End}\left(A, \mathcal{R}_{A}\right)$ be such that $\varphi(1)=0 \neq \varphi$. Then there exists some $i<\kappa$ such that $\varphi\left\lceil\operatorname{dom}\left(\sigma_{i}\right)=\sigma_{i}\right.$ and $c_{i}-a_{i} \in D_{i} \cap J_{i}$ but $\varphi\left(c_{i}-a_{i}\right)=\sigma_{i}\left(c_{i}-a_{i}\right)=$ $-\sigma_{i}\left(a_{i}\right)=-e_{i} \notin J_{i}$ and thus $\varphi \notin \operatorname{End}\left(A, \mathcal{R}_{A}\right)$. We conclude that for any $\varphi \in \operatorname{End}\left(A, \mathcal{R}_{A}\right)$ with $\varphi(1)=0$ we have $\varphi=0$, i.e. $\operatorname{End}\left(A, \mathcal{R}_{A}\right)=A$.

We construct a natural example to show that the algebraic hypothesis is needed.

ExAmPle 1. Let $L=\{f(x) / g(x) \mid f(x), g(x) \in \mathbb{Z}[x], g(x)$ primitive $\}$. Then $L$ is a commutative ring with free additive group and $\mathcal{R}_{L}=\{n L \mid$ $n \in \mathbb{N} \cup\{0\}\}$ is the set of all ideals of $L$. This means that $\operatorname{End}\left(L, \mathcal{R}_{L}\right)=$ $\operatorname{End}\left(L^{+}\right) \neq L$ and $L$ is not a Zassenhaus ring.

Proof. By Gauss' lemma, the set of all primitive integer polynomials is closed under multiplication, which implies that $L$ is a ring and each element of $L$ is an integer multiple of a unit in $L$. This shows that $\mathcal{R}_{L}$ is as stated, which implies $\operatorname{End}\left(L, \mathcal{R}_{L}\right)=\operatorname{End}\left(L^{+}\right) \neq L$. We will use Pontryagin's criterion [11, Theorem 19.1] to show that the countable group $L^{+}$is free abelian. (The argument is similar to the one used in [12].) Let $X$ be a finite subset of $L$. Then there exists some primitive polynomial $g$ and a 
finite subset $Y$ of $\mathbb{Z}[x]$ such that $X \subseteq g^{-1} Y$. Let $W$ be the pure subgroup of $\mathbb{Z}[x]$ generated by $Y$. Then $W$ is free, and so also is $X \subseteq g^{-1} W \cong W$. We have to show that $g^{-1} W$ is pure in $L$. Let $p$ be a prime, $f / h \in L$ and $b \in W$ such that $p f / h=b / g \in g^{-1} W$. This implies that $p g f=b h$. We read this equation modulo $p$ in the integral domain $(\mathbb{Z} / p \mathbb{Z})[x]$ to conclude that $b \equiv 0 \bmod p$ since the primitive polynomial $g \neq 0 \bmod p$. This shows that $b \in p \mathbb{Z}[x] \cap W=p W$ so that $p b^{\prime} / g=b / g$ and $b^{\prime} / g \in g^{-1} W$. Thus $X$ is contained in the pure, free subgroup $g^{-1} W$ of $L^{+}$. This proves that $L^{+}$is free.

Recall that if $R$ is a Zassenhaus ring, then $R$ is right rigid, that is, $\operatorname{End}\left(R, \mathcal{R}_{R}\right)=R$ as shown (for left rigidity) in [1]; see also [13, pp. 408410]. It is not clear when the converse holds. The following result can be found (implicitly) in [1]:

Theorem 4. Let $R$ be a ring such that $1 \in R$ and $R^{+}$is torsion-free and homogeneous of type zero. Let $\mathcal{F}$ be a family of at most countably many right ideals of the $\mathbb{Q}$-algebra $\mathbb{Q} R$ such that the following holds.

(1) $\operatorname{End}_{\mathbb{Q}}(\mathbb{Q} R, \mathcal{F})=\mathbb{Q} R$.

(2) $R^{+}=\sum \mathcal{F}$.

(3) $R^{+} /(X \cap R)$ is homogeneous of type zero for all $X \in \mathcal{F}$.

(4) For any prime integer $p$, the ring $R / p R$ has no non-zero nilpotent elements.

Then $R$ is a Zassenhaus ring.

3. Some rings due to A. L. S. Corner. First we generalize a construction due to Corner [8] (see also [13]). Let $\Lambda$ be a lattice with smallest element $1 \in \Lambda$ and $\mathcal{L}$ a family of sublattices of $\Lambda$ such that:

(a) $\Lambda=\bigcup_{L \in \mathcal{L}} L$

(b) $(\Lambda-L) \vee \mu \subseteq \Lambda-L$ for all $L \in \mathcal{L}$ and $\mu \in \Lambda$.

Condition (b) is equivalent to saying that if $\sigma \in \Lambda-L$ then $\sigma \vee \mu \notin L$ as well. Define $R=\bigoplus_{\lambda \in \Lambda} \lambda \mathbb{Z}$ and for $L \in \mathcal{L}$ let $R_{L}=\bigoplus_{\lambda \in L} \lambda \mathbb{Z}$. We define a multiplication on $R$ by setting $\lambda \cdot \mu=\lambda \vee \mu$ for all $\lambda, \mu \in \Lambda$. This turns $R$ into a commutative ring such that all $\lambda \in \Lambda$ are idempotent, and the minimal element 1 of the lattice becomes the $1 \neq 0$ of the ring. Obviously, $R_{L}$ is a subring of $R$ and $R=R_{L} \oplus N_{L}$ where $N_{L}=\bigoplus_{\lambda \in \Lambda-L} \lambda \mathbb{Z}$. Clause (b) implies that $N_{L}$ is an ideal of $R$ for all $L \in \mathcal{L}$. We define a topology $\mathfrak{T}$ on $R$ by employing the ideals $N_{L}, L \in \mathcal{L}$, as a basis of neighborhoods of 0 . Then $\mathfrak{T}$ is Hausdorff and each $R_{L}, L \in \mathcal{L}$, is discrete with respect to $\mathfrak{T}$ and $R$ becomes a topological ring. Let $\widehat{R}$ denote the completion of $R$. Then $\widehat{R} \subseteq \prod_{L \in \mathcal{L}} R / N_{L} \cong \prod_{L \in \mathcal{L}} R_{L}$ and $\widehat{R}=R_{L} \oplus \widehat{N}_{L}$ for all $L \in \mathcal{L}$. Moreover $\left(r_{L}+N_{L}\right)_{L \in \mathcal{L}} \in \widehat{R}$ if and only if $r_{L^{\prime}}-r_{L} \in N_{L}$ for all $L \subseteq L^{\prime} \in \mathcal{L}$. Consider 
the following family $\mathcal{F}$ of ideals of $\widehat{R}$ :

$\mathcal{F}=\left\{\lambda R_{L} \oplus \widehat{N}_{L}: \lambda \in L \in \mathcal{L}\right\} \cup\left\{(1-\lambda) R_{L} \oplus \widehat{N}_{L}: \lambda \in L \in \mathcal{L}\right\} \cup\left\{\widehat{N}_{L}: L \in \mathcal{L}\right\}$.

Our first goal is to show that $\operatorname{End}(\widehat{R}, \mathcal{F})=\widehat{R}$. To this end, let $\psi \in$ $\operatorname{End}(\widehat{R}, \mathcal{F})$ and $L \in \mathcal{L}$. Then $\widehat{R}=R_{L} \oplus \widehat{N}_{L}$ and with respect to that decomposition, we can write

$$
\psi=\left(\begin{array}{cc}
\alpha_{L} & 0 \\
\beta_{L} & \gamma_{L}
\end{array}\right)
$$

where $\alpha_{L}: R_{L} \rightarrow R_{L}, \gamma_{L}: \widehat{N}_{L} \rightarrow \widehat{N}_{L}$ and $\beta_{L}: R_{L} \rightarrow \widehat{N}_{L}$. Moreover, $\alpha_{L}\left(\lambda R_{L}\right) \subseteq \lambda R_{L}$ and $\alpha_{L}\left((1-\lambda) R_{L}\right) \subseteq(1-\lambda) R_{L}$ for all $\lambda \in L \in \mathcal{L}$. Let $\alpha_{L}(\lambda)=\sum_{\varrho \in L} \varrho z_{\varrho \lambda} \in R_{L}$ and note that $\lambda R_{L}=\bigoplus_{\lambda \leq \mu \in L} \mu \mathbb{Z}$. We infer that $z_{\varrho \lambda} \neq 0$ implies $\varrho \geq \lambda$, or equivalently, $z_{\varrho \lambda}=0$ whenever $\varrho \geq \lambda$.

Now consider $\alpha_{L}^{\prime}:=\alpha_{L}-\alpha_{L}(1)$. Then $\alpha_{L}^{\prime}(1)=0$ and, for all $\lambda \in L \in \mathcal{L}$, we have $\alpha_{L}^{\prime}\left(\lambda R_{L}\right) \subseteq \lambda R_{L}$ as well as $\alpha_{L}^{\prime}\left((1-\lambda) R_{L}\right) \subseteq(1-\lambda) R_{L}$, which means that $\alpha_{L}^{\prime}(-\lambda)=\alpha_{L}^{\prime}(1-\lambda) \in \lambda R_{L} \cap(1-\lambda) R_{L}=\{0\}$, since $\lambda, 1-\lambda$ is a pair of orthogonal idempotents in $R_{L}$. We infer $\alpha_{L}^{\prime}=0$, and thus for $a_{L}=\alpha_{L}(1) \in R_{L}$ we have $\alpha_{L}=a_{L}$. Now assume that $L \subseteq L^{\prime} \in \mathcal{L}$. Then $\psi\left\lceil R_{L^{\prime}}=a_{L^{\prime}} \in R_{L^{\prime}}\right.$ and $\psi\left\lceil R_{L}=a_{L} \in R_{L}\right.$. This implies that $a_{L^{\prime}}-a_{L} \in \widehat{N}_{L}$, i.e. $\left(a_{L}\right)_{L \in \mathcal{L}}$ is a Cauchy sequence in $\widehat{R}$. Since $\widehat{R}$ is complete, there is some $a \in \widehat{R}$ such that $a-a_{L} \in \widehat{N}_{L}$ for all $L \in \mathcal{L}$. Now consider $\psi^{\prime}=\psi-a$. Then $\psi^{\prime}(\widehat{R})=\psi^{\prime}\left(R_{L} \oplus \widehat{N}_{L}\right) \subseteq \widehat{N}_{L}$ since $\psi^{\prime}\left(R_{L}\right) \subseteq \widehat{N}_{L}$ and $\widehat{N}_{L} \in \mathcal{F}$ for all $L \in \mathcal{L}$. We conclude that $\psi^{\prime}(\widehat{R}) \subseteq \bigcap_{L \in \mathcal{L}} \widehat{N}_{L}=\{0\}$, and thus $\psi^{\prime}=0$ implies $\psi=a \in \widehat{R}$.

We claim that $\widehat{R}$ as well as $\widehat{R} / p \widehat{R}$ have no non-zero nilpotent elements for any prime $p$. Suppose that $a \neq 0$ is a nilpotent element. Then there is some $L \in \mathcal{L}$ such that $a=a^{\prime}+a^{\prime \prime}$ with $0 \neq a^{\prime} \in R_{L}$ and $a^{\prime \prime} \in \widehat{N}_{L}$. Then $a^{\prime}$ is nilpotent and we may assume that $a=a^{\prime} \in R_{L}$ and $a^{2}=0$. There is a finite subset $T$ of $L$ such that $a=\sum_{\varrho \in T} \varrho z_{\varrho}$ with $0 \neq z_{\varrho}$ in $\mathbb{Z}$ or $\mathbb{Z} / p \mathbb{Z}$. Then $0=a^{2}=\sum_{\mu} \mu\left(\sum_{\lambda \vee \varrho=\mu} z_{\lambda} z_{\varrho}\right)$. Let $\mu$ be a minimal element in the finite subset $T$ of $L$. Then $\lambda, \varrho \in T$ with $\lambda \vee \varrho=\mu$ minimal in $T$ implies $\lambda=\mu=\varrho$ and it follows that $z_{\mu}^{2}=0($ or $\equiv 0 \bmod p)$. This contradicts the assumption that $T \neq \emptyset$. We infer $a=0$.

Let $L \in \mathcal{L}$ and define

$$
\mathcal{F}_{L}=\left\{\lambda R_{L}: 1<\lambda \in L\right\} \cup\left\{(1-\lambda) R_{L}: 1<\lambda \in L\right\} .
$$

As the proof above shows, we have $\operatorname{End}\left(R_{L}, \mathcal{F}_{L}\right)=R_{L}$.

From now on we assume that $\Lambda$ as well as $\mathcal{L}$ are countable.

Let $P_{L, \lambda}^{0}$ and $P_{L, \lambda}^{1}$ be disjoint, infinite sets of primes and define

$$
T_{L, \lambda}^{i}=\left\langle p^{-1}: p \in P_{L, \lambda}^{i}\right\rangle
$$


a subgroup of $\mathbb{Q}$ for all $i=0,1$ and $\lambda \in L \in \mathcal{L}$. Let

$$
M_{L}=\sum_{\lambda \in L} T_{L, \lambda}^{0} \lambda R_{L}+\sum_{\lambda \in L} T_{L, \lambda}^{1}(1-\lambda) R_{L} \subseteq \mathbb{Q} R_{L} .
$$

As in the proof of Theorem 2 it is not hard to show that $\operatorname{End}\left(M_{L}\right)=R_{L}$, and we have:

The ring $R_{L}$ is a Zassenhaus ring.

Employing our countability hypothesis, we can also show that the ring $\widehat{R}$ is a Zassenhaus ring, but we also want to get a topological isomorphism between $\operatorname{End}(M)$ and $\widehat{R}$. To this end consider

$$
W=\bigoplus_{L \in \mathcal{L}} M_{L} \subseteq \bigoplus_{L \in \mathcal{L}} \mathbb{Q} R_{L} \cong \bigoplus_{L \in \mathcal{L}} \mathbb{Q}\left(\widehat{R} / \widehat{N}_{L}\right) .
$$

By the incompatibility of the types of the $M_{L}$, each $M_{L}$ is fully invariant in $W$. For each pair $\left(L, L^{\prime}\right) \in \mathcal{L} \times \mathcal{L}$ with $L \subset L^{\prime}$ we introduce some more distinct infinite sets of primes $P_{L, L^{\prime}}$ which are disjoint from all the others and define the corresponding subgroups $T_{L, L^{\prime}}$ of $\mathbb{Q}$. Set

$$
M=W+\sum_{L \subset L^{\prime} \in \mathcal{L}}\left(1_{L}+1_{L^{\prime}}\right) T_{L, L^{\prime}} \widehat{R}
$$

where $1_{L}$ is the identity in the $L$-coordinate of $W$. Let $\psi \in \operatorname{End}(M)$. Then $\psi\left(M_{L}\right) \subseteq M_{L}$ and $\psi \uparrow M_{L}=a_{L} \in R_{L} \cong \widehat{R} / \widehat{N}_{L}$. Moreover, $\psi\left(1_{L}+1_{L^{\prime}}\right)=$ $a_{L}+a_{L^{\prime}} \in\left(1_{L}+1_{L^{\prime}}\right) \widehat{R}$ for all $L \subset L^{\prime} \in \mathcal{L}$. It follows that $a_{L}-a_{L^{\prime}} \in \widehat{N}_{L}$ and $\left(a_{L}\right)_{L \in \mathcal{L}}$ is a Cauchy sequence whose limit we denote by $a \in \widehat{R}$, i.e. $a-a_{L} \in \widehat{N}_{L}$ for all $L \in \mathcal{L}$. This shows that $\operatorname{End}(M)=\widehat{R}$ is a topological isomorphism, i.e. the finite topology of $\operatorname{End}(M)$ is the same as the topology $\mathfrak{T}$ of $\widehat{R}$. We have shown:

THEOREM 5. Let $\Lambda$ be the countable lattice and $\mathcal{L}$ the countable family of sublattices of $\Lambda$, and $R$ be the commutative ring derived from the lattice as above. Then there exists an $\widehat{R}$-module $M$ such that $M \subseteq \bigoplus_{L \in \mathcal{L}} \mathbb{Q}\left(\widehat{R} / \widehat{N}_{L}\right)$ and $\operatorname{End}(M)$ with its finite topology is isomorphic as a topological ring to $\widehat{R}$ with its topology $\mathfrak{T}$.

In the proof of Theorem A in Corner [8, p. 254] (see also [13, p. 588, Theorem 15.1.2]) a $\operatorname{ring} R$ is constructed as follows. Let $I, J$ be sets of the same cardinality $\kappa$. Let $\Lambda$ be the lattice of all finite subsets of $I \times J$ and for each finite subset $\varrho$ of $I$ let $L_{\varrho}$ be the set of all finite subsets of $\varrho \times J$. Let $\mathcal{L}$ be the family of all these $L_{\varrho}$ 's. If, topologically, $\operatorname{End}(M)=\widehat{R}$, then each non-zero summand of $M$ is a direct sum of $\kappa$ summands. Setting $\kappa=\aleph_{0}$ we get

COROLlary 2. If we choose the ring $\widehat{R}$ as in the proof of Theorem $A$ in [8], then the countably generated $\widehat{R}$-module $M$ in Theorem 5 has the prop- 
erty that any non-zero summand of $M$ is a direct sum of countably many non-zero summands.

4. Corner's ring for the Leavitt theorem. We will study the left ideals of non-commutative rings whose original construction is due to A. L. S. Corner [7] with the aim of showing that they are Zassenhaus rings. This gives rise to their immediate realizations as endomorphism rings.

Let $q$ be a fixed positive integer, $[0, q]=\{i \mid i \in \mathbb{N}, 0 \leq i \leq q\}$ a corresponding interval and $R$ an integral domain such that $q R \neq R$. We consider the $R$-algebra $A$ freely generated by elements $\sigma_{i}, \sigma^{j}, i, j \in[0, q]$, subject only to the relations $\sigma^{i} \sigma_{j}=\delta_{i j}$ (the Kronecker delta) and $\sum_{i \in[0, q]} \sigma_{i} \sigma^{i}=1$. We call the elements in $[0, q]$ letters and let $W$ be the set of all words in these letters. If $u, v \in W$, then $u \wedge v$ is the concatenation of $u$ and $v$. For example, if $u=123$ and $v=045$, then $u \wedge v=123045$. We will use bold face letters (like $\mathbf{u}$ ) to denote elements in $W$. Moreover $\mathbf{u}^{+}$denotes the last letter in the word $\mathbf{u}$, so $123^{+}=3$, and $\sigma_{\mathbf{u}}=\sigma_{u_{1}} \ldots \sigma_{u_{k}}$ if $\mathbf{u}=u_{1} \ldots u_{k}$ with $u_{i} \in[0, q]$. The element $\sigma^{\mathbf{u}}$ is defined in the same manner. From [13, p. 591, (15.1.5)] it follows that $A$ is a free $R$-module with basis

$$
B=\left\{\sigma_{\mathbf{i}} \sigma^{\mathbf{j}}: \mathbf{i}, \mathbf{j} \in W, \mathbf{i}^{+} \neq 0 \text { or } \mathbf{j}^{+} \neq 0\right\} .
$$

If $\mathbf{u}=u_{1} \ldots u_{s}$, then we define $\mathbf{u}^{d}=u_{s} \ldots u_{1}$ by reversing the product. We have

$$
\sigma_{\mathbf{s}} \sigma^{\mathbf{t}} \sigma_{\mathbf{i}} \sigma^{\mathbf{k}}= \begin{cases}\sigma_{\mathbf{s}} \sigma^{\mathbf{t}_{0} \wedge \mathbf{k}} & \text { if } \mathbf{t}=\mathbf{t}_{0} \wedge \mathbf{i}^{d}, \\ \sigma_{\mathbf{s} \wedge \mathbf{i}_{0}} \sigma^{\mathbf{k}} & \text { if } \mathbf{i}=\mathbf{t}^{d} \wedge \mathbf{i}_{0}, \\ 0 & \text { otherwise. }\end{cases}
$$

If $\mathbf{t}=\mathbf{t}_{0} \wedge \mathbf{i}$ for some $\mathbf{t}_{0}$, we write $\mathbf{i} \leq_{r} \mathbf{t}$. If $\mathbf{i}=\mathbf{t} \wedge \mathbf{i}_{0}$ for some $\mathbf{i}_{0}$, we write $\mathbf{t} \leq_{\ell} \mathbf{i}$. For $\mathbf{k} \in W$, let $\mathbf{k}^{m}=\mathbf{k} \wedge \ldots \wedge \mathbf{k}$ denote the concatenation of $m$ copies of $\mathbf{k}$. Moreover, we adopt the convention that if $\mathbf{k}=\langle k\rangle \in W$ is a singleton, we write $k$ in place of $\mathbf{k}$. Let $W_{\mathbf{k}}=\left\{\mathbf{t} \in W: \mathbf{k} \not \leq_{r} \mathbf{t}\right\}$.

Claim 3. Let $F=\bigoplus_{i \in \omega} b_{i} \mathbb{Q}$ be a vector space over $\mathbb{Q}$ and $A_{n}=$ $\bigoplus_{i \in \mathbb{N}}\left(n b_{i-1}+b_{i}\right) \mathbb{Q}$ be subspaces for $n \in \omega$. Then $\bigcap_{n=0}^{\infty} A_{n}=\{0\}$ and $F=b_{0} \mathbb{Q} \oplus A_{n}$ for all $n \geq 0$.

Proof. Observe that $A_{n}=\bigoplus_{i=1}^{\infty}\left(-(-n)^{i} b_{0}+b_{i}\right) \mathbb{Z}$ and let $x=\sum_{i=0}^{m} b_{i} z_{i}$ in $A_{0}$ be such that $x \in A_{n}$. It follows that

$$
\sum_{i=0}^{m}(-n)^{i} z_{i}=0 \text {. }
$$

Now we assemble the equations $(1),(2), \ldots,(m+1)$ to obtain a homogeneous system of linear equations with a Vandermonde determinant; we infer that all $z_{i}=0$ and thus $x=0$. 
Claim 4. Let $A$ be the free $R$-algebra as above. Then

$$
\bigcap_{n=0}^{\infty} \mathbb{Q} A\left(n+\sigma^{\mathbf{k}}\right)=\{0\} \quad \text { for all } \mathbf{k} \in W
$$

and each $A\left(n+\sigma^{\mathbf{k}}\right)$ is a direct summand of $A^{+}$.

Proof. Note that $A \sigma^{\mathbf{k}}=\left\langle\sigma_{\mathbf{s}} \sigma^{\mathbf{t} \wedge \mathbf{k}}: \mathbf{s}, \mathbf{t} \in W\right\rangle$ and let $b_{\mathbf{s}, \boldsymbol{\tau}, j}=\sigma_{\mathbf{s}} \sigma^{\boldsymbol{\tau} \wedge \mathbf{k}^{j}}$ where $\mathbf{t} \wedge \mathbf{k}=\boldsymbol{\tau} \wedge \mathbf{k}^{j}$ and $\boldsymbol{\tau} \in W_{\mathbf{k}}=\left\{\boldsymbol{\beta} \in W: \mathbf{k} \not \not_{r} \boldsymbol{\beta}\right\}$. Then

$$
\mathbb{Q} A \sigma^{\mathbf{k}}=\bigoplus_{\mathbf{s} \in W} \bigoplus_{\boldsymbol{\tau} \in W_{k}}\left(\bigoplus_{j=1}^{\infty} b_{\mathbf{s}, \boldsymbol{\tau}, j} \mathbb{Q}\right) \text {. }
$$

We finally consider

$$
A\left(n+\sigma^{\mathbf{k}}\right)=\left\langle n \sigma_{\mathbf{s}} \sigma^{\mathbf{t}}+\sigma_{\mathbf{s}} \sigma^{\mathbf{t} \wedge \mathbf{k}}\right\rangle=\bigoplus_{\mathbf{s} \in W} \bigoplus_{\boldsymbol{\tau} \in W_{k}}\left(\bigoplus_{j=0}^{\infty}\left(n b_{\mathbf{s}, \boldsymbol{\tau}, j}+b_{\mathbf{s}, \boldsymbol{\tau}, j+1}\right) \mathbb{Z}\right)
$$

and apply the previous claim to get our conclusion.

Next we prove

Claim 5. Let $k \in[0, q]$ be a letter and $B_{k}=A\left(\sigma^{k}+\sigma_{\mathbf{i}}\right)$. Then $\mathbb{Q} B_{k} \cap$ $\mathbb{Q} B_{k^{\prime}}=\{0\}$ for $k \neq k^{\prime} \in[0, q]$ and each $B_{k}$ is a direct summand of $A^{+}$.

Proof. Note that

$$
\begin{aligned}
A\left(\sigma^{k}+\sigma_{\mathbf{i}}\right)= & \left\langle\sigma_{\mathbf{s}} \sigma^{\mathbf{t} \wedge k}+\sigma_{\mathbf{s}} \sigma^{\mathbf{t}} \sigma_{\mathbf{i}}: \mathbf{s}, \mathbf{t} \in W\right\rangle \\
= & \left\langle\sigma_{\mathbf{s}} \sigma^{\boldsymbol{\tau} \wedge \mathbf{i}^{d} \wedge k}+\sigma_{\mathbf{s}} \sigma^{\boldsymbol{\tau}}: \mathbf{s}, \boldsymbol{\tau} \in W\left(\text { i.e. } \mathbf{i}^{d} \leq_{r} \mathbf{t}\right)\right\rangle \\
& \oplus\left\langle\sigma_{\mathbf{s}} \sigma^{\boldsymbol{\tau} \wedge k}: \boldsymbol{\tau} \in W, \mathbf{i}^{d}, \boldsymbol{\tau} \text { are } \leq_{r} \text {-incomparable }\right\rangle \\
& \left.\oplus\left\langle\sigma_{\mathbf{s}} \sigma^{\mathbf{t} \wedge k}+\sigma_{\mathbf{s} \wedge \mathbf{j}}: \mathbf{i}^{d}=\mathbf{j}^{d} \wedge \mathbf{t}, \mathbf{s} \in W \text { (i.e. } \mathbf{t} \leq_{r} \mathbf{i}^{d}\right)\right\rangle .
\end{aligned}
$$

The rest is easy to see by inspection.

Let $\psi \in \operatorname{End}\left(A^{+}\right)$be such that $\psi(1)=0$ and $\psi(X) \subseteq X$ for all left ideals $X$ of $A$. Then $\psi\left(\sigma^{\mathbf{k}}\right)=\psi\left(n+\sigma^{\mathbf{k}}\right) \in \bigcap_{n=0}^{\infty} A\left(n+\sigma^{\mathbf{k}}\right)=\{0\}$ and we have $\psi\left(\sigma^{\mathbf{k}}\right)=0$. Moreover, $\psi\left(\sigma_{\mathbf{i}}\right)=\psi\left(\sigma^{k}+\sigma_{\mathbf{i}}\right) \in B_{k}$ for all $0 \leq k \leq q$ and since $B_{k} \cap B_{k^{\prime}}=0$ for $k \neq k^{\prime}$, we infer $\psi\left(\sigma_{\mathbf{i}}\right)=0$. Now consider $\psi\left(\sigma_{\mathbf{i}} \sigma^{\mathbf{k}}\right)=\psi\left(n \sigma_{\mathbf{i}}+\sigma_{\mathbf{i}} \sigma^{\mathbf{k}}\right)=\psi\left(\sigma_{\mathbf{i}}\left(n+\sigma^{\mathbf{k}}\right)\right) \in A\left(n+\sigma^{\mathbf{k}}\right)$ for all $n \geq 0$, which implies that $\psi=0$ as desired.

Define

$$
\mathcal{F}_{P}=\left\{A\left(n+\sigma^{\mathbf{k}}\right) \mid n \in P, \mathbf{k} \in W\right\} \cup\left\{A\left(\sigma^{k}+\sigma_{\mathbf{i}}\right) \mid 0 \leq k \leq q, \mathbf{i} \in W\right\}
$$

where $P$ is any infinite set of primes. What we have shown so far is the following:

Lemma 2. With the above notations, $\operatorname{End}\left(A, \mathcal{F}_{P}\right)=A$.

A few minor modifications of the above arguments also yield 
Corollary 3. If $\mathcal{F}_{P}^{\prime}=\left\{\mathbb{Q} X: X \in \mathcal{F}_{P}\right\}$ is the corresponding family of ideals in $\mathbb{Q} A$, then also End $_{\mathbb{Q}}\left(\mathbb{Q} A, \mathcal{F}_{P}^{\prime}\right)=\mathbb{Q} A$.

We fix the following

Notation. Let $R$ be a torsion-free ring with $1 \in R$ such that $R^{+}$is homogeneous of type zero.

(1) Let $\mathcal{F}$ be a family of at most countably many left ideals of $R$ such that each $V \in \mathcal{F}$ is a pure subgroup of $R^{+}$and $R^{+} / V$ is homogeneous of type zero for all $V \in \mathcal{F}$.

(2) Let $\mathcal{F}_{\mathbb{Q}}=\{\mathbb{Q} V: V \in \mathcal{F}\}$, a family of left ideals of the $\mathbb{Q}$-algebra $\mathbb{Q} R$, such that $\operatorname{End}\left(\mathbb{Q} R, \mathcal{F}_{\mathbb{Q}}\right)=\mathbb{Q} R$.

(3) Let $\prod=\left\{P_{V}: V \in \mathcal{F}\right\}$ be a family of infinite, disjoint sets of prime numbers. Let $T_{V}=\left\langle 1 / p: p \in P_{V}\right\rangle$ for all $V \in \mathcal{F}$, a subgroup of $\mathbb{Q}$. Moreover, let $\tau_{V}$ denote the type of $T_{V}$.

(4) Define $M=R+\sum_{V \in \mathcal{F}} T_{V} V \subseteq \mathbb{Q} R$.

(5) Let $M\left(\tau_{V}\right)$, as usual, denote the subgroup of $M$ of all elements of type larger than or equal to $\tau_{V}$; see e.g. [14].

We will prove the following using these notations.

LEMma 3. We have $M\left(\tau_{V}\right)=V_{*}$, the purification of $V$ in $M$ for all $V \in \mathcal{F}$. Assume that for all $v \in V \in \mathcal{F}$ and $p \in P_{V}$ the inclusion $v V \subseteq p R$ implies that $v \in p R$. Then $\operatorname{End}_{\mathbb{Z}}(M)=R$.

Proof. Let $V \in \mathcal{F}$ and $m^{\prime} \in M\left(\tau_{V}\right)$. Then there is some $k \in \mathbb{N}$ such that $m=k m^{\prime} \in R \cap M\left(\tau_{V}\right)$. Let $P=\left\{p \in P_{V}: p^{-1} m \in M\right\}$. Then $P$ is a cofinite subset of $P_{V}$ and $p^{-1} m \in t_{p}(M / R)=\left(p^{-1} V+R\right) / R$ and thus $m \in \bigcap_{p \in P} V+p R$; it follows that $m+V \in M / V$ has type $\tau_{V}$ and so $m \in V$. Thus $m^{\prime} \in V_{*}$. This shows that $\mathbb{Q} V \cap M=V_{*}=M\left(\tau_{V}\right)$. Now let $\varphi \in \operatorname{End}(M)$ and $\psi \in \operatorname{End}(\mathbb{Q} R)$ its unique extension, i.e. $\psi\lceil M=\varphi$. Since $\varphi\left(M\left(\tau_{V}\right)\right) \subseteq M\left(\tau_{V}\right)=V_{*}$ we see that $\psi(\mathbb{Q} V) \subseteq \mathbb{Q} V$ for all $V \in \mathcal{F}$. By $(2)$ we have $\psi \in \mathbb{Q} R$ and $w=\psi(1) \in M$, which implies that $w M \subseteq M$. We may assume that $w=v / p \in M-R$ for some $v \in V$ and $p \in P_{V}$ and we have $v p^{-2} V \subseteq M$ but $p\left(t_{p}(M / R)\right)=0$. Thus $v p^{-1} V \subseteq R$ and hence $v V \subseteq p R$. Thus, by our hypothesis, $v \in p R$, a contradiction. We conclude that $w \in R$ as required.

TheOREM 6. Let $q \geq 2$ be a natural number and $A$ the ring constructed above. Then there exists $A \subseteq M \subseteq \mathbb{Q} A$ such that $\operatorname{End}\left(M^{+}\right)=R$. It follows that $\bigoplus_{n} M \cong \bigoplus_{m} M$ if and only if $m \equiv n \bmod q$.

Proof. We may assume that $P \cap \bigcup_{V \in \mathcal{F}} P_{V}=\emptyset$. All we need to show in order to apply Lemma 2 is that $v V \subseteq p A$ for some $v \in V \in \mathcal{F}_{P}$ implies that $v \in p A$. This follows easily by inspection. The last assertion follows as in [6]; see also [11, Theorem 91.6]. 


\section{References}

[1] J. Buckner and M. Dugas, Quasi-localizations of $\mathbb{Z}$, Israel J. Math. (2007), to appear. [2] -, 一, Left-rigid rings, J. Algebra, to appear.

[3] M. C. R. Butler, A class of torsion-free rings of finite rank, Proc. London Math. Soc. 15 (1965), 680-698.

[4] -, On locally free torsion-free rings of finite rank, J. London Math. Soc. 43 (1968), 297-300.

[5] A. L. S. Corner, Every countable reduced torsion-free ring is an endomorphism ring, Proc. London Math. Soc. (3) 13 (1963), 687-710.

[6] -, On a conjecture of Pierce concerning direct decompositions of abelian groups, in: Proc. Colloq. Abelian Groups (Tihanyi, 1963), Akadémiai Kiadó, Budapest, 1964, 43-48.

[7] —, Additive categories and a theorem of W. G. Leavitt, Bull. Amer. Math. Soc. 75 (1969), 78-82.

[8] - On the existence of very decomposable abelian groups, in: Abelian Group Theory (Honolulu 1982), Lecture Notes in Math. 1006, Springer, Berlin, 1983, 354-357.

[9] P. Eklof and A. Mekler, Almost Free Modules, Elsevier, Amsterdam, 2002.

[10] P. Eklof and S. Shelah, The Kaplansky test problems for $\aleph_{1}$-separable groups, Proc. Amer. Math. Soc. 126 (1998), 1901-1907.

[11] L. Fuchs, Infinite Abelian Groups, Vols. 1 \& 2, Academic Press, New York, 1970, 1973.

[12] R. Göbel and D. Herden, Constructing sharply transitive $R$-modules of rank $\leq 2^{\aleph_{0}}$, J. Group Theory (2007), to appear

[13] R. Göbel and J. Trlifaj, Approximation Theory and Endomorphism Algebras, de Gruyter, Berlin, 2006.

[14] A. Mader, Almost Completely Decomposable Groups, Algebra Logic Appl. 2, Gordon \& Breach, Amsterdam, 2000.

[15] H. Zassenhaus, Orders as endomorphism rings of modules of the same rank, J. London Math. Soc. 42 (1967), 180-182.

Department of Mathematics

Baylor University

Waco, TX 76798, U.S.A.

E-mail: Manfred_Dugas@baylor.edu
Fachbereich Mathematik Universität Duisburg-Essen 45117 Essen, Germany E-mail: Ruediger.Goebel@uni-due.de

Received 28 August 2006;

in revised form 8 March 2007 